\title{
ELABORACIÓN DE PROGRAMAS COMPUTACIONALES MEDIANTE LENGUAJES GRÁFICOS A PARTIR DE LOGARITMOS SUGERIDOS POR APLICACIÓN DE MAPAS CONCEPTUALES
}

\author{
Plamen Neichev Nechev \\ Profesor asociado de la Universidad Pedagógica Nacional \\ Mónica Eilene Valencia $D$. \\ Estudiante Lic, en Física Universidad Pedagógica Nacional monicval@usa.net
}

\begin{abstract}
This article attempts to initiate a thorough review on the relationship between the concept of map theory and the strategy for using computers in physics laboratories as a measuring tool and to help high school students in programming steps for virtual instruments measuring. The problem to be solved and the definition of map are discussed in the introduction, while the relationship between the constructivist method, the concept map and the graphic programming are described in the following para graphs. In the conclusion part, the achievements will be pointed out.
\end{abstract}

\section{RESUMEN}

El presente artículo pretende poner en marcha un estudio exhaustivo sobre la relación entre la teoría de los mapas conceptuales y la estrategia para el uso del computador como herramienta de medición en el laboratorio de física, con el fin de ayudar a los estudiantes de secundaria en la etapa de programación de los instrumentos virtuales de medición. El problema por solucionar y la definición de mapa conceptual se tratan en la introducción, mientras que la relación entre el método constructivista, los mapas conceptuales y la programación gráfica se desarrollan en los párrafos siguientes. En la conclusión se enumeran los alcances hasta ahora obtenidos.

\section{INTRODUCCIÓN}

Es bien sabido que las destrezas manuales son habilidades adquiridas a través de un adiestramiento y no son los productos buscados durante el proceso de educación en física. Luego, al realizarse un laboratorio en la escuela secundaria, el principal problema no consiste en la ejecución correcta de las mediciones pertinentes y ni siquiera en la construcción mecánica de cielos elementos para el equipo de medición ya que es preciso afirmar que los principales retos del trabajo experimental son: la madurez de poder diseñar las prácticas y el conocimiento para tratar con los inevitables errores cometidos al ejecutarse un laboratorio. Sin embargo, la carencia de base instrumental en nuestras secundarias y la falta de tiempo suficiente para poder diseñar los equipos de medición y/o su composición para la clase de laboratorio son definitivas al momento de decidirse qué tipo de actividades se desarrollarán durante la experiencia. En tal sentido, lo que domina el panorama educativo es el ofrecimiento por parte del profesor de un laboratorio donde el método es el 'único' posible mientras que la ejecución es automática. Como resultado, las clases de laboratorios de física en la secundaria se vuelven aburridas, ineficientes y por ende pierden el sentido para el cual fueron previstas. 
La realidad de a situación anterior ha provocado muchas discusiones, investigaciones y propuestas. Teniendo en cuenta los avances de los últimos tiempos en la informática, uno de los autores P.N. ha realizado una propuesta para utilizar el computador como la principal herramienta en el laboratorio de física escolar. Con este fin, se contempla la aplicación del producto llamado kit básico educativo de la empresa NATIONAL INSTRUMENTS, el cual consiste en una tarjeta de adquisición de datos, con sus conectores respectivos $\mathrm{y}$, por supuesto, el software especifico desarrollado para este propósito. El último, entre otras cosas, usa un lenguaje de programación gráfico (lenguaje G) Ilamado LabVIEW de las primeras letras o sílabas de su nombre completo Laboratory Virtual Instrument Engineering Workbench. La estrategia del uso del computador con ayuda de interfaces estándar y lenguajes gráficos se ha abierto camino bajo el nombre de laboratorios por computador". Por otra parte. un lenguaje G es el diagrama de flujo del programa presentado en forma para ser ejecutado directamente por el computador sin la mediación de un código (como por ejemplo, esto ocurre durante la elaboración de un programa en el lenguaje $\mathrm{C}$ ). No obstante, la enorme facilidad al utilizarse el software LabVIEW (que es lo que nos permite pensar en la aplicación de éste en la secundaria), persiste el problema de ¿cómo comenzar y cuál será la manera más eficiente de cumplir con la tarea del diseño del laboratorio? Ahora bien, puesto que en una serie de colegios el uso de los mapas conceptuales no es una novedad demasiado exigente, es razonable plantear la siguiente inquietud: ¿de qué manera los conocimientos y las habilidades adquiridas con el manejo de tales mapas nos pueden llevar a un método para elaborar programas gráficos a través de LabVIEW de NATIONAL INSTRUMENTS?

La elaboración de mapas conceptuales permite que el estudiante llegue a tener conciencia del su propio proceso cognitivo o metacognición como bien afirma A. Ontoria en el libro Mapas Conceptuales. El desarrollo de tales actividades durante la realización de laboratorios por computador (claro, antes de la elaboración de un programa en términos de un lenguaje G) es muy importante, ya que no solo posibilita la síntesis de los datos observados y el análisis cualitativo completo de la situación, sino que permite al maestro conocer la forma como sus estudiantes interpretaron el fenómeno. Además, como "los conceptos son, según Novak, (...), las imágenes mentales que provocan en nosotros las palabras o signos '(Ontoria, Ballesteros, Cuevas, Giraldo, Martín, Molina, Rodríguez y Velez, 1996), está claro que un concepto, es decir, objetos y procesos, puede ser plasmados por medio de dibujos y, por ello, se puede establecer una estrecha relación entre los mapas conceptuales y el lenguaje gráfico o lenguaje $G$ de programación.

La noción del mapa conceptual, hasta donde los autores han podido indagar, aparece primero en los trabajos de Novak y Gowin a mediados de los años ochenta. Como consecuencia de una rápida aceptación, surgen propuestas y desarrollos tanto en las distintas áreas del conocimiento como en los distintos grupos de países con diferentes grados de desarrollo y tendencias culturales. En tal sentido, en el ámbito de la Educación en Física y para la región latinoamericana debemos mencionar los aportes de Marco Antonio Moreira. A nivel nacional, y enfocándonos en los desarrollos del tema, dados en la Universidad Pedagógica Nacional, debemos mencionar el trabajo de Pérez R. y Gallego-Badillo R. En realidad, uno de los autores, P.N. se familiarizó con las aplicaciones de los mapas conceptuales, durante el proceso de ciertas colaboraciones con algunos profesores del departamento de química de esta universidad.

El mapa conceptual se puede definir de muchas formas de acuerdo con la aplicación que se desee del mismo, sin embargo, existe una característica fundamental que lo diferencia de otros tipos de esquemas, y es que permite a los estudiantes hacer una organización jerárquica de los conceptos que se mezclan en un tema, relacionándolos 
entre sí por expresiones llamadas conectores. En consecuencia, según el mismo Novak y como lo menciona A. Ontoria (Qntoria, Ballesteros, Cuevas, Giraldo, Martín, Molina, Rodríguez y Velez, 1996), tenemos varias definiciones para lo que habitualmente se denomina mapa conceptual. Es decir, el mapa conceptual es:

1. Una estrategia: "Procuraremos poner ejemplos de experiencias sencillas, pero poderosas en potencia, para ayudar a los estudiantes a aprender y para ayudar a los educadores a organizar los materiales que se van a aprender'(Novak y Gowin, 1988, p. 19);

0

2. Un método: "La construcción de mapas conceptuales (...), que es un método para ayudar a los estudiantes y educadores a captar el significado de los materiales que se van a aprender" (Ibid.);

0

3. Un recurso: 'Un mapa conceptual es un recurso esquemático para representar un conjunto de significados conceptuales incluidos en una estructura de proposiciones' (Ibid.).

Sin embargo, nosotros en esta ocasión vamos a restar importancia a las diferencias entre las distintas definiciones, ya que en realidad son complementarias y las ideas que nos sugieren respecto de la elaboración de programas coinciden. Más aun, con tal propósito la existencia y el uso de otros nombres para representaciones gráficas del conocimiento, donde básicamente cambia el método de presentación, pero no la esencia de la jerarquización para el esquema cognitivo, no serán relevantes como algo esencialmente nuevo. Además, es de esperar que en un futuro no tan lejano, al llegarse a un desarrollo más unificado entre los esquemas en cuestión, es decir, los cuales ponen jerarquía dentro de los conceptos científicos manejados por la educación secundaria, será posible decantar una definición más rigurosa y carente de ambivalencias

Según Roger Anderson "el acto de recordar es visto como un proceso de acomodación y de modificación de la información existente en la memoria para optimizar el valor adaptativo de la respuesta en relación con la organización interna de las ideas y las demandas externas del entorno" (Andersen, Demetrius, 1993). Eso significa que, como se afirma más adelante en el mismo trabajo, durante el proceso de elaboración y mejoramiento del mapa conceptual las relaciones entrelazadas, o sea al mismo nivel o desde abajo hacia arriba, se enriquecen y al final llevan a la elaboración completa del esquema. Los autores por sus propias experiencias saben que durante el proceso de elaboración de un diagrama de flujo, característico para un leguaje gráfico, como por ejemplo LabVIEW de NATIONAL INSTRUMENTS, existen muchas semejanzas con la etapa de perfeccionamiento de un mapa conceptual. Entonces, la propuesta consiste en la utilización de la experiencia adquirida gracias al trabajo con mapas conceptuales para el aprendizaje de la programación gráfica.

\section{CONSTRUCTIVISMO Y MAPAS CONCEPTUALES}

El constructivismo ha sido una de las propuestas educativas más revolucionarias a lo largo de la historia, ya que pone al estudiante como el pilar central dentro del proceso 
educativo y en consecuencia considera la educación en sí misma, más como una "negociación conceptual" entre el maestro y el estudiante, que como una imposición unilateral de los conocimientos del primero sobre el segundo. Hay que reconocer, sin embargo, que este proceso de "negociación" es algo coordinado, puesto que el alumno, por lo menos a nivel secundaria o pre-grado, en realidad no inventa por sus propios medios la ciencia como tal. Ante las afirmaciones en cuestión surgen varias preguntas: ¿de qué manera se puede conocer lo que el alumno "sabe" o lo que ya ha elaborado?, ¿cómo facilitar al estudiante una exposición coherente y nutrida por sus ideas? y ¿cómo permitir que el educando verifique y modifique sus propias ideas con base en debates y experiencias? Dichas inquietudes no son de ninguna forma nuevas y los pedagogos desde los años setenta han sido enfrentados a estos problemas. Una de las respuestas, que a lo largo de las décadas se viene fomentando por los investigadores, y la que la consideramos como la más viable, fue hecha por Josef Novak mediante el desarrollo de una propuesta, consistente en la utilización de mapas conceptuales para el aprendizaje significativo. La idea es ver el mapa conceptual como 'instrumento para representar las tramas conceptuales o preposicionales" y además, como afirma él mismo "construir mapas conceptuales, (...), nos proveyó "aprender a aprender" (Porlan, Garcia y Canal 1995). Es así, como, dentro de la metodología constructivista el mapa conceptual cobra especial relevancia, porque refleja la organización conceptual que realiza cada estudiante en el proceso de aprendizaje; además su reelaboración permite visualizar los cambios conceptuales, que a través del tiempo, la percepción y asimilación de nuevas ideas provocan en los estudiantes, y, como afirman, R. Pérez y R. Gallego, sirve de herramienta para la "confrontación y el análisis de las formas de pensar entre los alumnos; entre los alumnos y profesor; y entre el grupo y la información proporcionada" (PérezyGallegoBadillo, 1995). En este sentido, las facilidades que proporciona la técnica de los mapas conceptuales para un aprendizaje constructivista, consideradas desde la perspectiva del espíritu de la programación gráfica y gracias a ciertas analogías entre esta y el método de los mapas conceptuales, se pueden plasmar en los ambientes informáticos específicos, los cuales sirven como base en el desarrollo del trabajo experimental en laboratorio de física al usar-se el computador en calidad de herramienta principal durante las actividades prácticas.

\section{LOS DIAGRAMAS DE FLUJO DE LOS LENGUAJES GRÁFICOS APLICADOS A PROCESOS DE MEDICION}

El punto de partida de la propuesta es concebir un diagrama de flujo elaborado para el lenguaje gráfico LabVIEW 5.1 como una especie de reflejo de un mapa conceptual. Considerando el presente intento como la aproximación "cero" en el proceso iterativo que acompaña cualquiera investigación seria, se pueden dejar al lado, por ahora, las cuestiones más avanzadas y teóricas, como por ejemplo las semejanzas y diferencias en las propiedades topológicas de los mapas conceptuales y los diagramas de flujo utilizadas en el caso de programación gráfica. Lo importante es ¿cómo a partir de un mapa conceptual programar una experiencia utilizando lenguaje $\mathrm{G}$ ?

Veamos un parangón entre el mapa conceptual y un programa escrito en LabVIEW. Existen las siguientes afinidades:

- Tanto el mapa conceptual como el diagrama de flujo contienen nodos. En el caso del mapa conceptual estos son los conceptos que se construyen y en el caso de la programación gráfica son diferentes comandos o subrutinas. 
- En ambos casos los conectares son líneas de organización y subordinación en dirección de arriba hacia abajo y de izquierda a derecha.

- Los bucles en el programa corresponden a contornos cerrados en el mapa.

- Los condicionales expresados por el lenguaje $\mathrm{G}$ corresponden a un nodo conceptual donde las líneas salientes pertenecen al mismo nivel jerárquico.

Para fijar ideas, veamos el mapa conceptual del método de medición estándar que se aplica en los laboratorios de física. Sobre el proceso de medición se puede apreciar que:

- Bajo ninguna circunstancia podemos conocer el valor "exacto" de una magnitud física, ya que los errores son inevitables y siempre persisten en el resultado de una única medición.

- Por tal motivo, es necesario realizar varias mediciones para tener el valor medio de estas, el cual se muestra como el valor más probable de dicha magnitud.

- Finalmente, se establece un intervalo de confianza determinado por el valor medio y el error estándar.

El algoritmo de medición se puede presentar en el siguiente mapa conceptual:

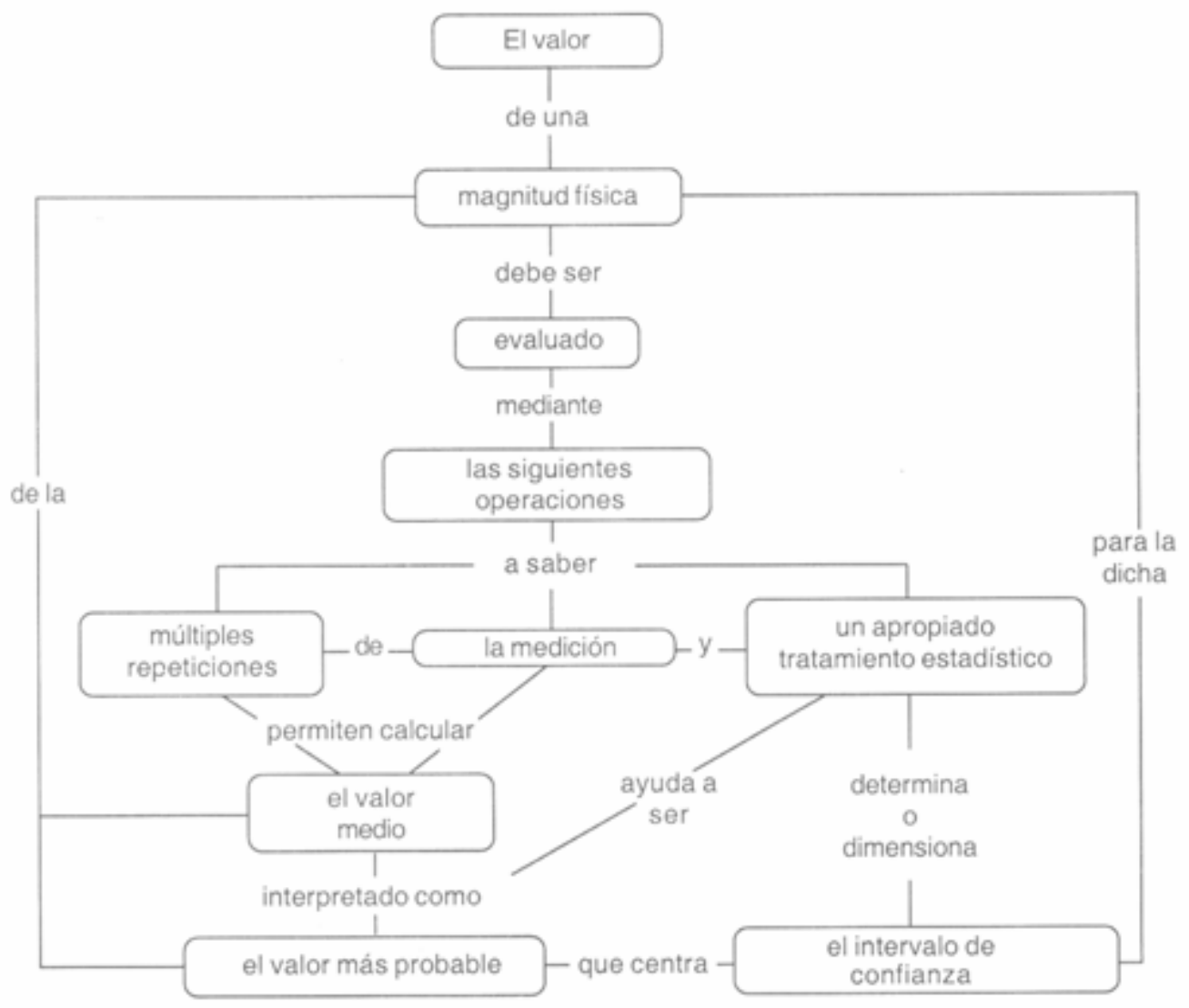

Es de notar que siempre las líneas que terminan en la parte superior de los nados relacionan los últimos como conceptos subordinados. También las líneas horizontales que van de un nodo a otro subordinan el nodo derecho al izquierdo, mientras que las lineas que conectan en forma lateral conceptos de diferente jerarquía enriquecen el significado 
de los conceptos principales. Por tal motivo y teniendo en cuenta las expresiones conectares, no es necesaria una indicación explícita del orden jerárquico.

Veamos ahora el diagrama de flujo de un programa gráfico cuya elaboración fue sugerida por el mapa conceptual considerado. En éste se utiliza una subrutina de medición sencilla y el interrogante en el nodo significa que la medición única puede ser de cualquier tipo (por ejemplo de voltaje, intensidad de corriente, temperatura, etc.). EI símbolo de la subrutina corresponde al concepto central en nuestro mapa llamado "la medición”, alrededor del cual se desarrolla todo el proceso de obtención de los valores más probables de la magnitud.

El bucle de ejecución de mediciones múltiples corresponde al contorno en forma de triángulo, cuya parte superior contiene varios (en nuestro caso dos) conceptos de jerarquía horizontal. El otro triángulo, cuya base horizontal es el lado inferior del mapa "no genera" ningún bucle en LabVIEW 5.1, ya que un bucle involucra el concepto de repeticiones antes de que sea ejecutada la acción concreta.

Las entradas y salidas del programa, como es de esperar, no tienen su análogo en el mapa conceptual ya que por sí solas son detalles del lenguaje. Sin embargo, las entradas son la información "faltante", o no especificada a propósito en el mapa conceptual, lo cual permite un uso múltiple del mapa. Las salidas son la información esperada después de haberse cumplido la "misión" del mapa conceptual.

El condicional y por supuesto, el diálogo relacionado con el no, es relevante puesto que su función es puramente técnica relacionada con la grabación o no de los resultados.

Finalmente, los cálculos del valor medio y el error estándar se hacen por subrutinas incrustadas en lenguaje LabVIEW 5.1 y son presentados en el diagrama de flujo en la parte superior derecha.

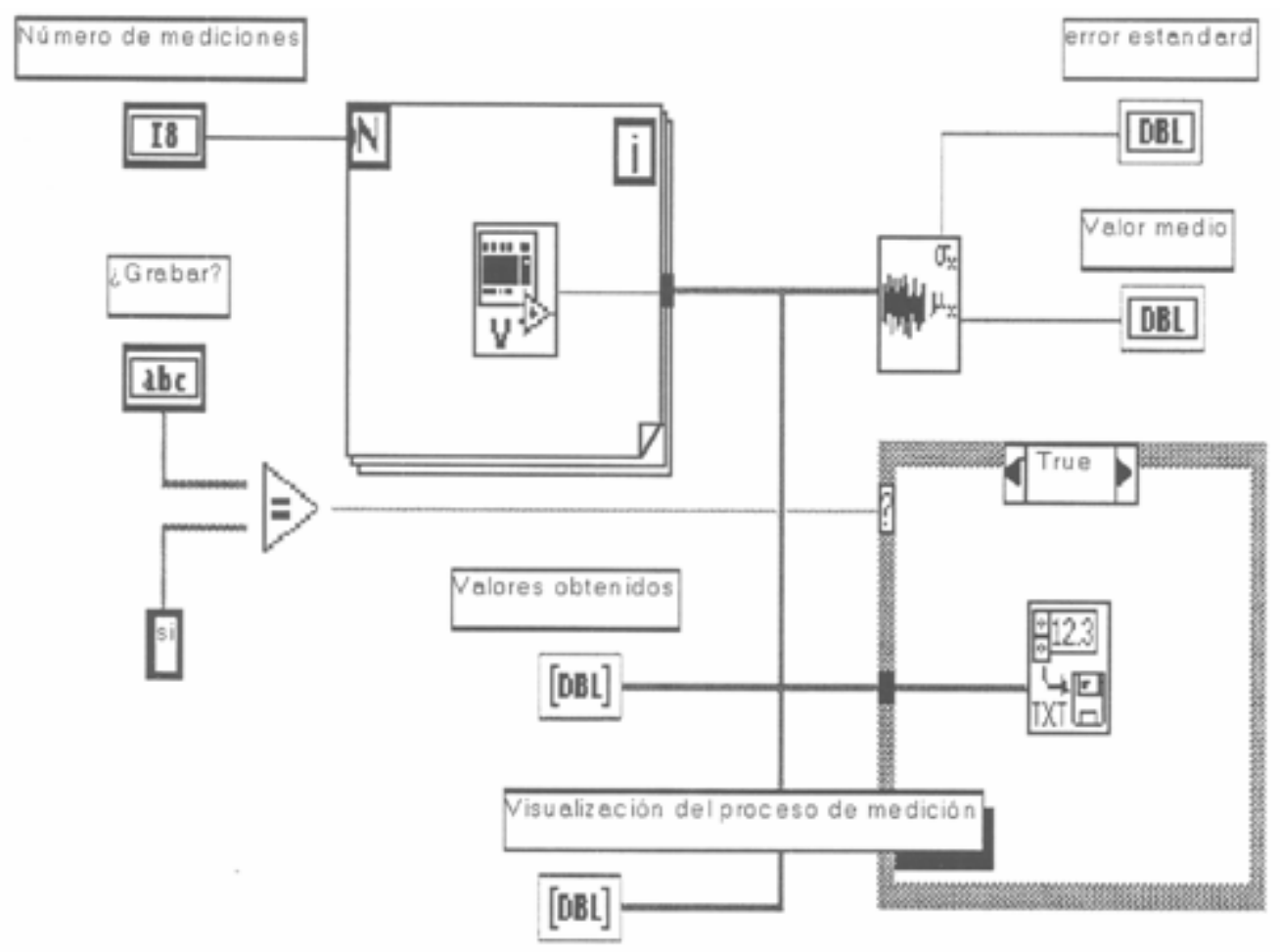




\section{CONCLUSIONES Y PROPUESTAS}

Se pueden hacer las siguientes conclusiones:

Existen semejanzas en los fundamentos de un mapa conceptual y un diagrama de flujo para el leguaje $G$ de LabVIEW que permiten plasmar las características de aprendizaje constructivista de los mapas conceptuales a las prácticas de programación gráfica.

Los procesos repetitivos presentan una topología clara en el dibujo del mapa conceptual la cual se puede traspasar sin dificultades a la ventanilla de programación del LabVIEW 5.1

La jerarquía de un mapa conceptual es prácticamente la misma que la de los lenguajes gráficos.

A manera de sugerencia anotemos que así como el concepto "la medición", el cual ocupa la parte central del mapa conceptual, permite iniciar su elaboración, el símbolo de medición (la interrogante) sirve como punto de inicio para la elaboración del programa.

\section{REFERENCIAS}

Anderson O. R. and Demetrius O. J. (1993). Journal of Reseach in Science Teaching, 30, 953-969.

Markham K. M.; Mintzes J. J. and Jones M. G. (1994). Journal of Reseach in Science Teaching, 31, 91-101.

Ontoria A.; Ballesteros A.; Cuevas C.; Giralda L.; Martín I.; Molina A.; Rodríguez A. y Vélez U. (1996). Mapas conceptuales una técnica para aprender. Madrid: Narcea.

Parlan R.; García J. E. y Canal P. (1995). Constructivísmo y enseñanza de las ciencias. Sevilla: Diada.

Pérez, R.y Gallego Badillo R. (1995). Corrientes constructivistas. Bogotá: Magisterio.

Ruiz Primo M. A. and Shavelson R. J. (1996). Journal of Reseach in Science Teaching, 33, 569-600. 